\title{
Laminar-Turbulent Boundary Layer Transition Imaging Using IR Thermography
}

\author{
Brian K. Crawford, Glen T. Duncan Jr., David E. West, William S. Saric \\ Department of Aerospace Engineering, Texas A\&M University, College Station, USA \\ Email: crawford.briank@tamu.edu
}

Received May 1, 2013; revised June 1, 2013; accepted June 8, 2013

Copyright (C) 2013 Brian K. Crawford et al. This is an open access article distributed under the Creative Commons Attribution License, which permits unrestricted use, distribution, and reproduction in any medium, provided the original work is properly cited.

\begin{abstract}
Experimental techniques for imaging laminar-turbulent transition of boundary layers using IR thermography are presented for both flight and wind tunnel test environments. A brief overview of other transition detection techniques is discussed as motivation. A direct comparison is made between IR thermography and naphthalene flow visualization. A technique for obtaining quantitative transition location is presented.
\end{abstract}

Keywords: IR Thermography; Laminar; Turbulent; Transition; Flight Test; Wind Tunnel; Fluid Flow

\section{Introduction}

Over the past several years, laminar flow research has been ongoing at the Texas A\&M Flight Research Laboratory (FRL) using both the Swept Wing In Flight Testing (SWIFT) and Swept Wing In Flight Testing Excrescence Research (SWIFTER) models. Both models were designed to be mounted to the hardpoint of a Cessna O2A Skymaster aircraft, and use IR thermography as the primary measurement technique for laminar-turbulent transition imaging. Figures $\mathbf{1}$ and $\mathbf{2}$ show SWIFTER mounted under the port wing. SWIFT has the same outer dimensions and is mounted on the same pylon when it is flown.

IR thermography exploits the fact that when there exists a temperature differential between the surface of an airfoil and the ambient fluid, the turbulent region will tend to equalize to the ambient temperature faster due to its higher shear stress. This difference in temperature can be imaged using IR thermography [1-3]. The two models differ in how they achieve this differential. SWIFT is cooled by cold soaking at $3200 \mathrm{~m}$ MSL until it equalizes to ambient temperature. The aircraft then descends rapidly through warmer air. SWIFTER, on the other hand, has an internal heating sheet that is used to warm the surface above ambient. As such, SWIFTER does not require a high-altitude cold soak. Additionally, since SWIFTER does not rely on an externally applied temperature differential, it is also capable of utilizing IR thermography when installed in a wind tunnel which operates under adiabatic conditions. An example of SWIFTER in the Klebanoff-Saric Wind Tunnel (KSWT) is shown in Figure 3.

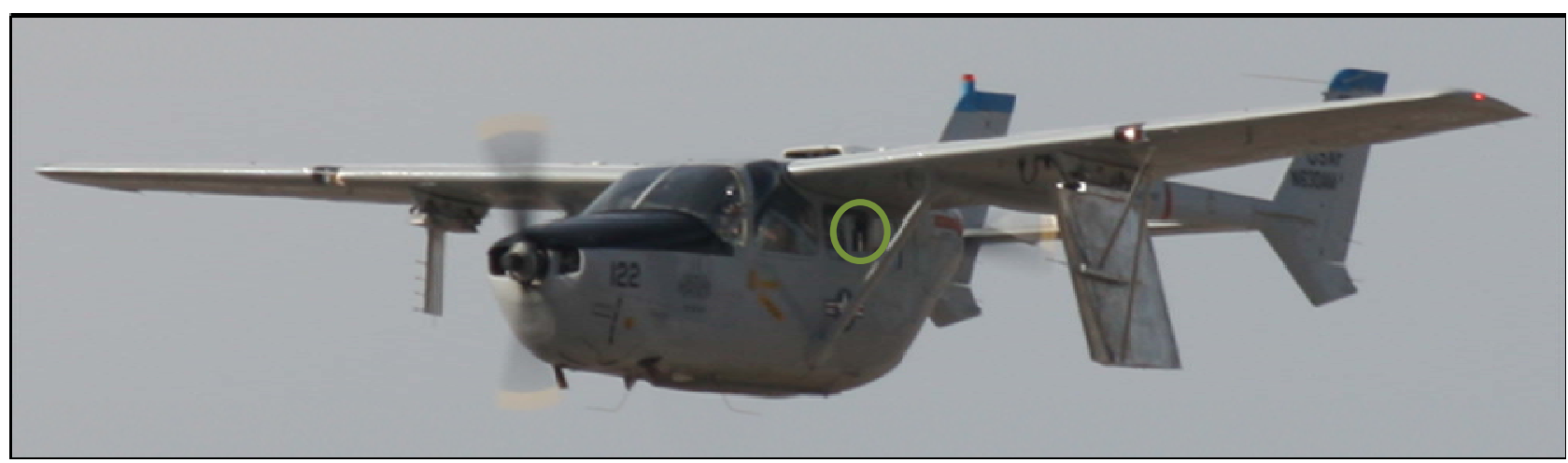

Figure 1. Cessna O-2A Skymaster with SWIFTER on the port outboard hardpoint with the IR camera port circled in green. 


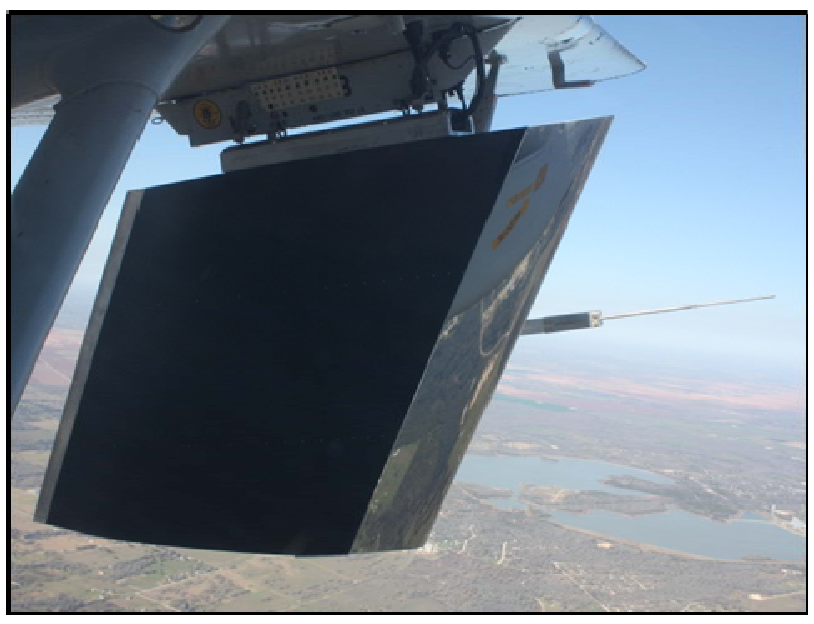

Figure 2. SWIFTER as viewed from the pilot.

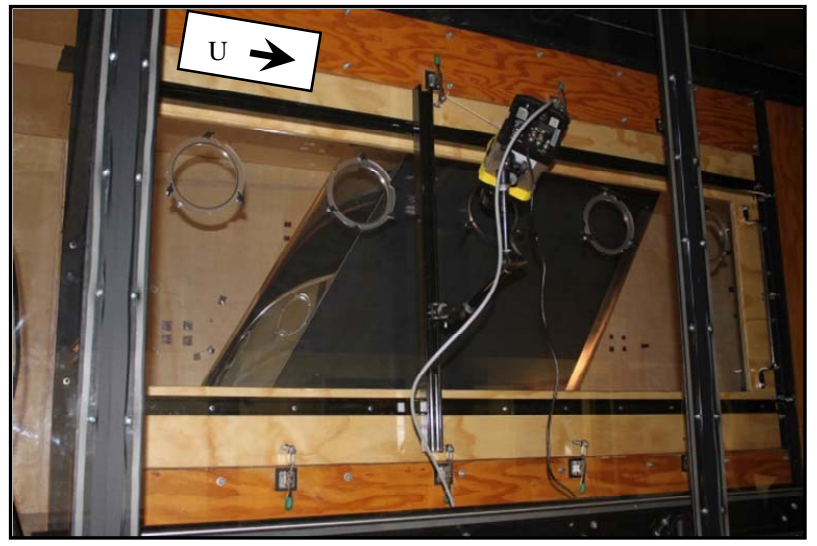

Figure 3. SWIFTER Mounted in the KSWT along with the SC8100 IR Camera.

\section{Motivation}

Laminarization of a significant portion of a transport aircraft wing would result in an estimated overall drag savings of $15 \%$ [4]. This would result in considerable fuel savings for any such aircraft; however, in order to make reliable laminar flow a reality, it must be studied and quantified in a laboratory environment.

Several techniques for laminar-turbulent transition detection have been developed and implemented over the last several decades. Some of the more common techniques include hot-film anemometry, surface-mounted microphones, sublimation based flow visualization, shear sensitive paint, and oil-film interferometry. A review of options for laminar-turbulent transition is given in [5].

Hot-film anemometry provides local shear stress measurements. These local shear stress measurements can be used to detect whether flow is laminar or turbulent over the sensor as shown in [6]. However, to image an entire transition front, many sensors are needed. This becomes unfeasible when mm accuracy is desired.
Surface-mounted microphones measure local pressure fluctuations. Turbulent flow can be detected by a large rise in the broadband oscillations in pressure. However, much like hot-films, the number of microphones required to image an entire transition front to sub centimeter accuracy becomes infeasible.

Sublimation based flow visualization involves applying a coating of naphthalene or similar chemical to the surface under test and allowing the chemical to sublimate off during the experiment. The turbulent region sublimates much faster than the laminar region, and as such, the resulting pattern of remaining naphthalene indicates what part of the flow is laminar. This technique can provide excellent images, but requires complete sublimation and re-application for every test point. Reference [7] provides examples of this technique in a flight environment.

For oil film interferometry, multiple drops of oil are placed on the model. When the model is brought up to speed, the oil shears proportionally with the local shear stress. The shearing of the oil is then measured optically, and the variation in shear can be used to detect regions of laminar vs. turbulent flow. The down side is that this technique relies on conditions being constant for the duration of the test, and must be re-applied for every test. Additionally, oil residue can pose an issue in a clean tunnel environment. Further details on this technique can be found in [8].

Shear sensitive paint can be used to image shear stress over the entire surface. However, shear sensitive paints that are capable of measuring relatively low shear stress are very fragile, and must be re-applied somewhat frequently. As shear sensitive paint technology improves, this may become a practical technique for imaging laminar-turbulent transition in many environments. Reference [9] provides more information on the use of shear sensitive paint.

In summary, most existing techniques for boundary layer transition have limited fidelity, as is the case with hot-films and surface microphones or require extended time on very stable conditions, naphthalene flow visualization and oil film interferometry. In order to detect transition fronts rapidly with high fidelity, a technique such as infrared thermography is required.

\section{Theory}

Because of differences in shear stress, laminar and turbulent boundary layers have markedly different convection coefficients. As such, a heated surface will cool much faster under the influence of a turbulent boundary layer than under a laminar boundary layer; visa-versa for a cooled surface.

This can be exploited experimentally by utilizing a heated or cooled substrate with a thin, insulating exterior coating. Since the coating is heated or cooled at a rea- 
sonably uniform rate from below by the substrate, but is under the influence of a very discontinuous convection rate from above, a strong temperature gradient is developed at the point of transition. This gradient can then be imaged via the use of an IR camera. A notional diagram of this process is shown in Figure 4.

\section{Experimental Setup}

As far as IR thermography is concerned, the experiment requires three main components; an IR camera, a heated or cooled model, and a suitable thin, insulating coating on the model.

\subsection{Cooled Model}

SWIFT is constructed from milled aluminum. The model itself has no specific provisions for cooling. Instead, the model is cold soaked at $3200 \mathrm{~m}$ MSL until the IR image shows that it has equalized to the local atmospheric temperature. This typically takes around 20 minutes. Once the model is cool, the aircraft then rapidly descends through the warmer air at lower altitudes. At a 300 - 600 $\mathrm{m} / \mathrm{min}$ descent rate, the standard lapse rate of the atmosphere is sufficient to induce the necessary temperature differential. However, the necessary descent rate will vary based on model construction and local atmospheric
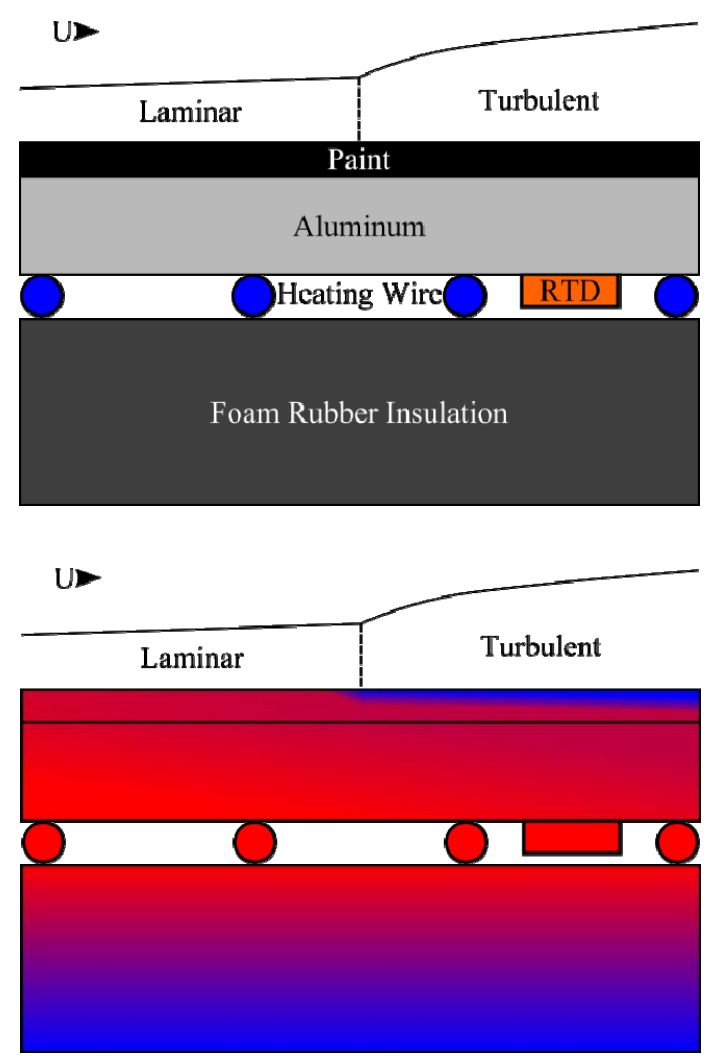

Figure 4. Construction of heated model (not to scale) (top) Temperature distribution near transition (bottom). conditions. Reference [10] contains addition information about the SWIFT model.

\subsection{Heated Model}

SWIFTER is also constructed from milled aluminum. Due to structural considerations, the model has two distinct regions of wall thicknesses; a forward and aft region. In the forward region, the skin is between $12.7 \mathrm{~mm}$ and $25.4 \mathrm{~mm}$. In the aft region, the skin is $3.2 \mathrm{~mm}$ thick. Thus, the heating sheet for the model is split into two regions, one forward and one aft, which run on separate control loops in order to keep the two regions matched in temperature. The forward and aft heating sheets have a maximum power of $200 \mathrm{~W}\left(792 \mathrm{~W} / \mathrm{m}^{2}\right)$ and $300 \mathrm{~W}\left(350 \mathrm{~W} / \mathrm{m}^{2}\right)$, respectively.

The heating sheet itself was constructed using presheathed heating wire. This particular wire is typically used to warm tile floors in houses from below. The heating wire is bonded onto the inside of the test surface of the model via RTV silicone, as shown in Figure 5. In the forward region, the wire is bonded in at $12.7 \mathrm{~mm}$ spacing, while the aft region is spaced at $25.4 \mathrm{~mm}$. It is covered by a layer of $12.7 \mathrm{~mm}$ polyurethane foam to help direct as much of the heat as possible through the test surface.

The temperature controller is connected to a pair of surface mounted resistance temperature detectors (RTDs) placed on the inside surface of the test section inside each of the two heating sheets. There is also a reference RTD placed on the inside of the unheated side of the model. During a test, the controller is generally set to drive the test side RTDs to $5^{\circ} \mathrm{C}$ higher than the reference RTD. Since these measurements are on the inside of the model, they do not match the external surface temperatures exactly. However, they do maintain a surface temperature that is consistent between the two regions, and nominally a constant offset from the atmospheric temperature. For this imaging technique, the exact tempera-

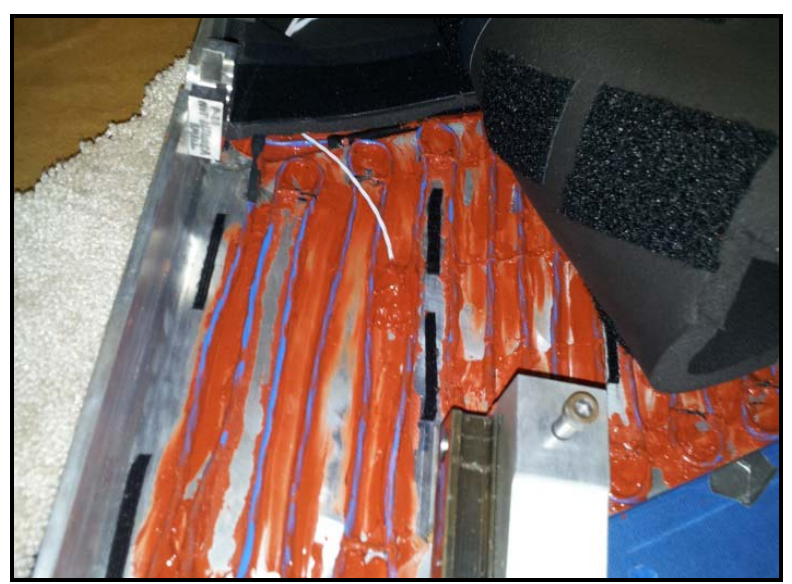

Figure 5. Heating wire (blue) bonded into SWIFTER using RTV (red) with the insulation (black) pulled back. 
ture offset is not important, as long as the model is a few degrees warmer than ambient. Additional details about the SWIFTER model can be found in [11].

\subsection{IR Camera}

Early experiments with SWIFT utilized a FLIR SC3000 IR camera. The SC3000 has a $320 \times 240$ pixel sensor with a maximum frame rate of $60 \mathrm{~Hz}$. It operates in the 8 - 9 micron wavelength band.

Later experiments with SWIFT, and all experiments with SWIFTER utilize a FLIR SC8100 IR camera. The SC8100 has a $1024 \times 1024$ pixel sensor capable of sampling at $132 \mathrm{~Hz}$. It has a temperature resolution of better than $25 \mathrm{mK}$, and operates in the 3 - 5 micron wavelength band. For these experiments, the frame rate is typically set to $20 \mathrm{~Hz}$ in order to reduce data overhead. Additionally, the system does not respond rapidly enough to warrant a higher frame rate. Figure 6 shows a comparison between the two cameras.

In the flight environment, the camera is mounted facing outwards through an opening in the rear port window, as seen just above the port wing strut in Figure 1. In the
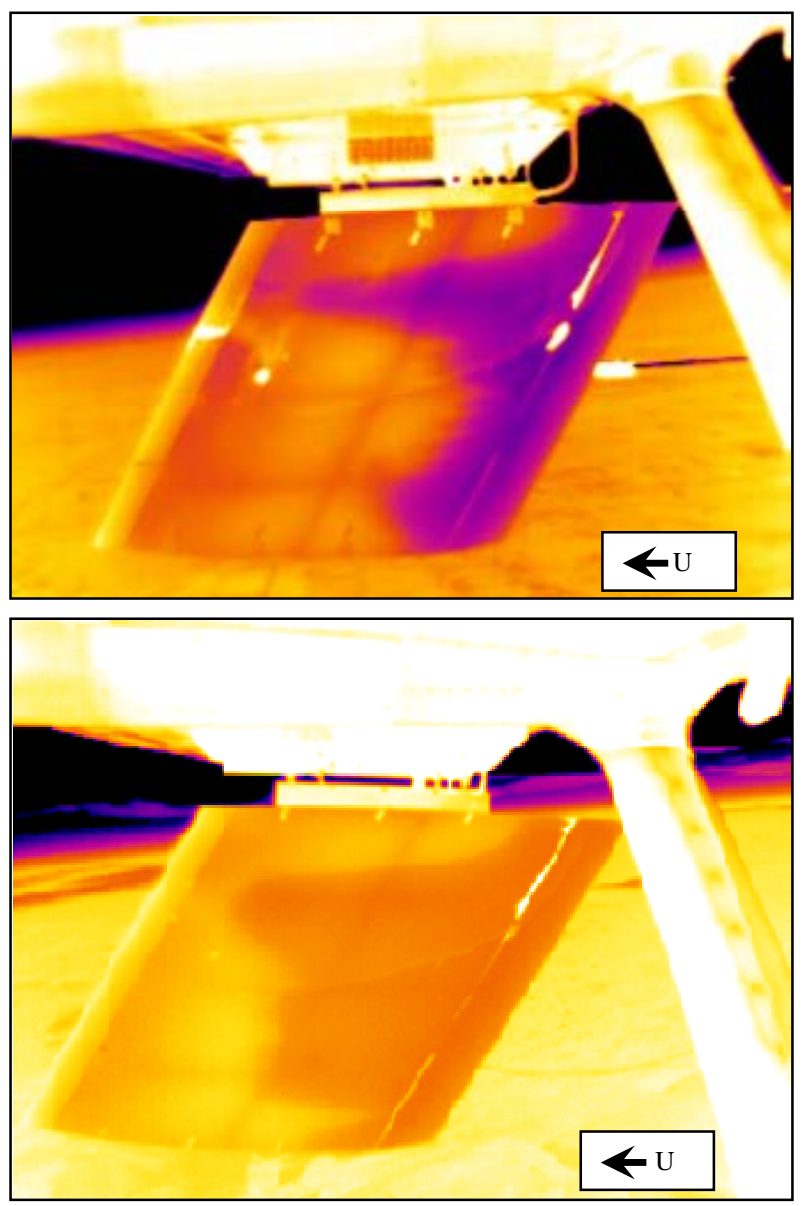

Figure 6. SWIFT using the SC8100 w/17 mm lens (top) vs. the SC3000 w/17 mm lens (bottom). wind tunnel, the camera is mounted facing through an opening in the test section viewing window, as shown in Figure 3. In both cases, an opening in the window is utilized rather than an IR transparent window. This is due to the substantial cost of an IR transparent window large enough to fit the camera lens.

\subsection{Paint Selection}

As mentioned previously, the model must be coated with a thin insulating layer in order to hold a strong temperature gradient. Additionally, the coating must have as low of a reflectivity in the IR band as possible. This is particularly important in flight where the exhaust flare from the front engine and the warm earth reflect brightly in the image otherwise. In order to meet these parameters, Sherwin Williams F93 mil-spec aircraft paint in lusterless black is currently used on SWIFTER.

SWIFT has a flat black powder coated surface applied over aluminum. This coating holds a gradient well, but is fairly reflective in the IR band.

Initially SWIFTER had a flat black powder coat as well. However, that particular coating was not thick enough to hold a strong temperature gradient at the surface; as such, the images were completely washed out. Additionally, the powder coat was very reflective in the IR band. The new aircraft paint is very flat in the IR band. The new paint was applied over the existing powder coat to a total thickness on the order of 300 microns. The thicker paint results in a much crisper image.

\section{Results}

IR thermography has been used in three principle configurations at the TAMU FRLand KSWT. The first configuration used SWIFT as a cooled flight model. This was followed recently by SWIFTER as a heated flight model as well as a heated wind tunnel model.

\subsection{Cooled Flight Model (SWIFT)}

Cooling the model via cold soaking, as discussed in Section 4.1, results in images such as shown in Figures 6 and 7 , where the laminar region is cooler (darker), and the turbulent region is warmer (lighter). Figure 6 shows a comparison between the SC8100 and the SC3000. The extra resolution is capable of showing finer details of the transition front. When the $50 \mathrm{~mm}$ lens is installed on the SC8100 and aimed at the lower forward region, as shown in Figure 7, even more detail is apparent, specifically a much finer wedge structure. In all SWIFT images, one spanwise and three chordwise darker regions are visible. These correspond to the internal structural members of the model, which do not heat up as quickly as the skin. Additionally, there is a pair of curved lines corresponding to the reflection of the fuselage and radio antenna in 


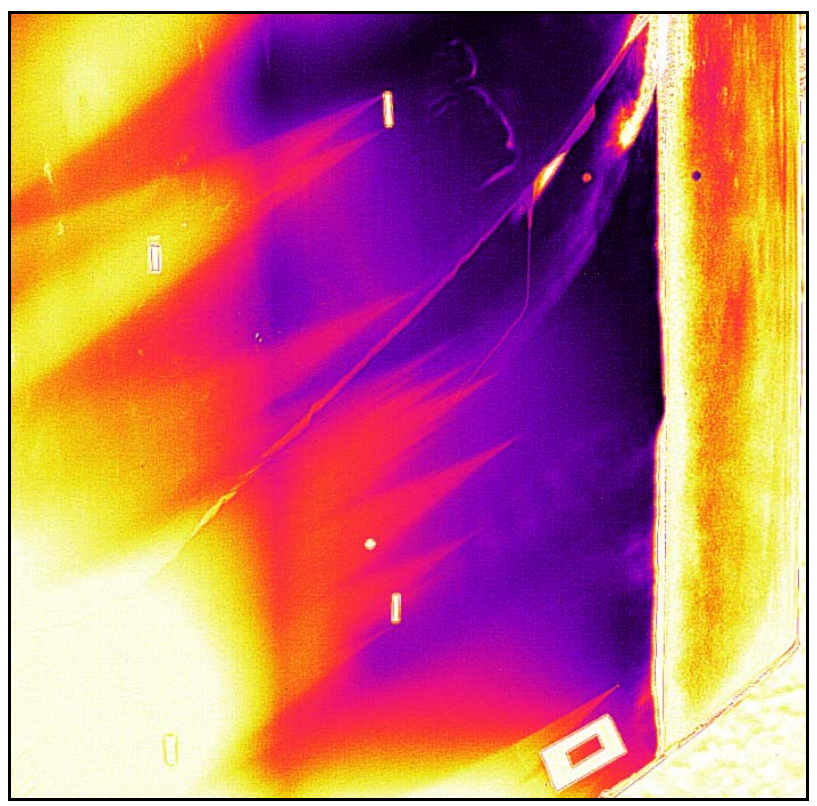

Figure 7. SWIFT using the SC8100 w/50 mm lens.

the model. This is due to the reflectivity of the particular powder coat.

The primary advantage to cooling a model via highaltitude cold soak is that it does not require any addition equipment or coatings on the model, other than a suitable paint job. However, every set of tests requires a 20 minute cold soak. Given a typical descent rate during a test, a high speed descent from $3200 \mathrm{~m}$ to $900 \mathrm{~m}$ allows for around 5 - 10 test points before the model must be cold soaked again depending on atmospheric conditions. For a typical sortie with an O-2A Skymaster, two dives are practical, resulting in 10 - 20 test points per flight.

\subsection{Heated Flight Model (SWIFTER)}

Actively heated models do not share this disadvantage. For a heated model, a high speed descent is only necessary in order to hit high speed test points. Additionally, no appreciable recycle time is required between dives. As such, 6 dives of 5 - 10 test points are typical. This results in 30 - 60 test points per flight. The only real disadvantage is that the extra complexity of a heating sheet must be integrated into the model. However, compared to the cost of flight time saved by increased testing efficiency, this additional start-up cost is quickly recovered. An example image is shown in Figure 8. Note that when the model is heated, the image coloration is inverted compared to the cooled case. In Figure 8, the vertical seam between the forward and aft regions is visible due to transient effects.

\subsection{Heated Wind Tunnel Model (SWIFTER)}

Since SWIFTER can be mounted in the wind tunnel, it
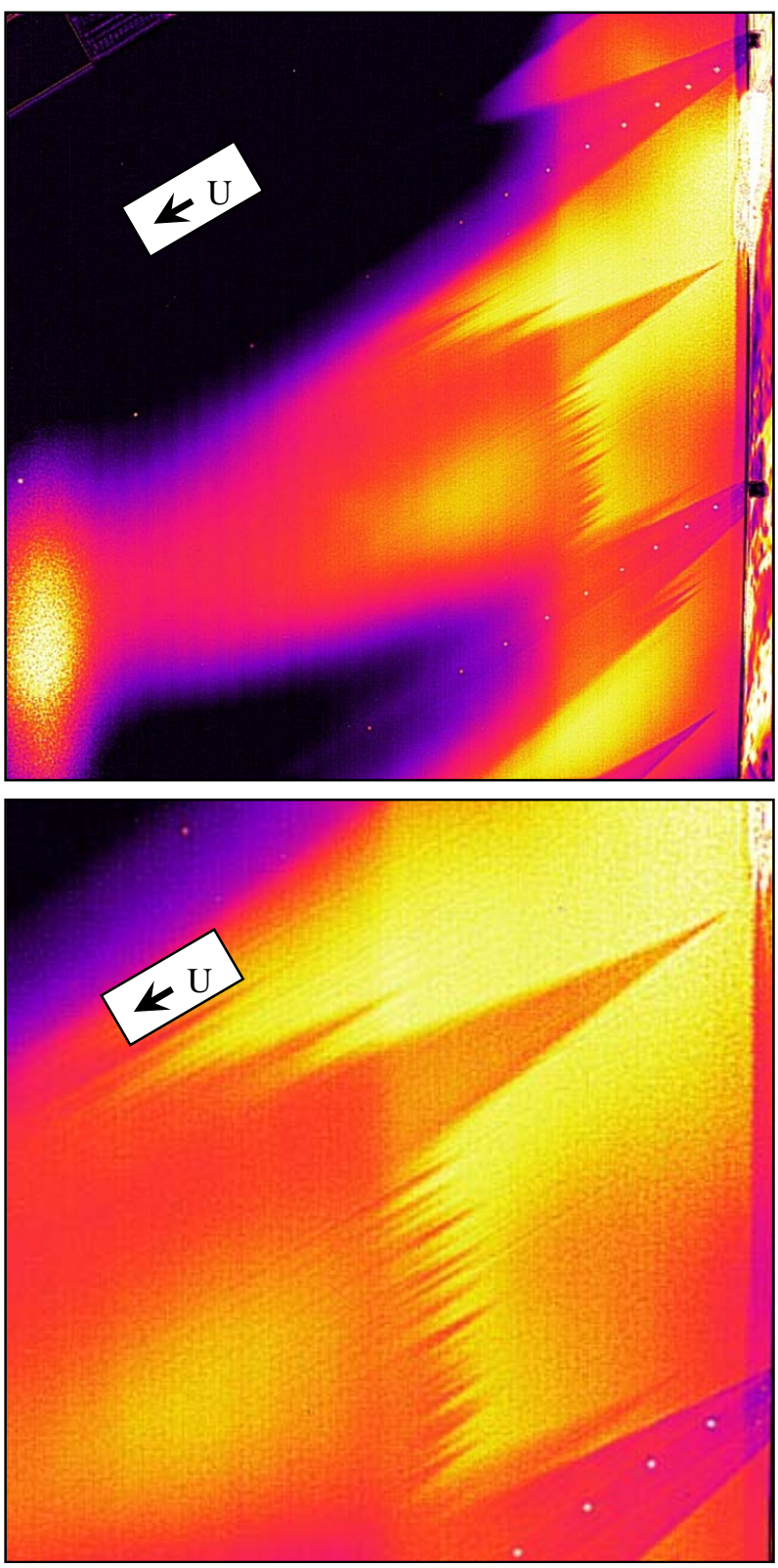

Figure 8. SWIFTER using the SC8100 w/ 50mm lens Full image (top), and zoomed in on mid-span (bottom).

provides an opportunity to compare IR thermography to the more ubiquitous naphthalene flow visualization. Naphthalene performs well at resolving fine structures in the flow, which IR thermography has not generally been able to match. However, given a sufficiently high resolution camera, such as the SC8100 and the appropriate choice of coating, comparable results can be obtained, as shown in Figure 9. The fine streaking in the forward region is clearly visible in both images. One of the disadvantages to naphthalene is that it is very susceptible to the uniformity of the coating applied, as shown by the waviness in the structures shown in the image. Additionally, the main motivating benefit of IR thermography is 

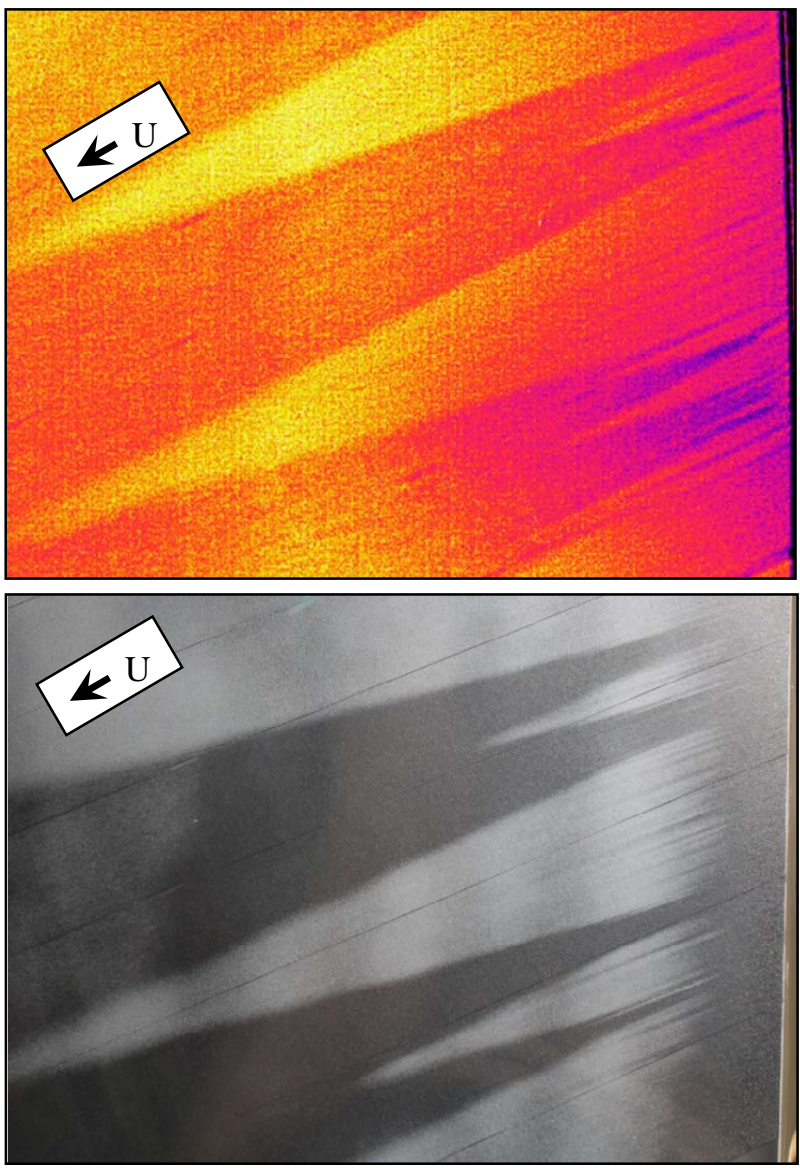

Figure 9. SWIFTER transition fronts in the wind tunnel at a repeated condition using IR thermography (top), and naphthalene flow visualization (bottom).

its quick turnaround time. When the transition front changes, the image responds in under a second, while naphthalene would require a complete re-application. Because of this fact, an IR thermography test can easily capture over 200 test points in an hour, while a typical day of naphthalene runs involves only 3 test points.

\subsection{Post-Processing of Transition Images}

Once images have been captured via IR thermography, the next step is to process them into quantitative data. The top panel of Figure 10 shows a typical IR image as filtered and colorized by FLIR's Examine-IR software. The outlined area is the region of interest for the experiment. The bottom panel shows the image after coordinate trans- formation, transition detection, and filtering.

In order to generate this post processed image, the raw data file is first converted into temperature values, and dead pixels are corrected. Any remaining hot or cold pixels are then corrected in turn. The columns of the image are then normalized with respect to their neighbors in order to remove a large portion of the sensor noise inherent to the construction of this particular type of cam-

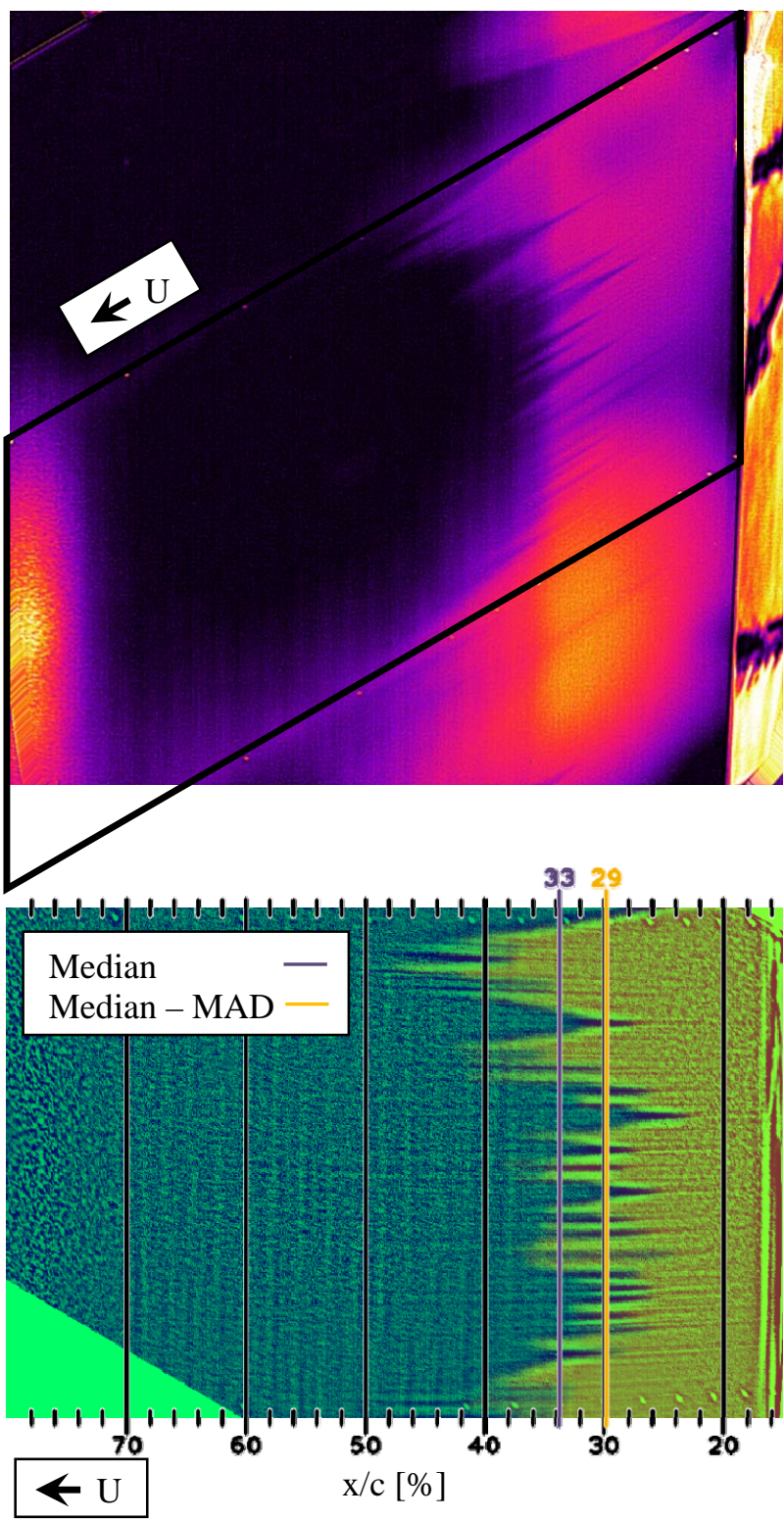

Figure 10. FLIR filtered image (top) compared to postprocessed image (bottom).

era. This also corrects for the temperature differential between the forward and aft regions mentioned earlier. These processes result in a reasonably clean image, which is then cropped and transformed to make vertical lines constant in chord and horizontal lines constant in span. The image is then duplicated into a pair of images. The second image is then spatially low-passed and subtracted from the first. This filtering removes any slowvarying features such as structural members, non-uniform heating due to internal electronics and aircraft exhaust flare. The resulting image is shown as the green channel in the final image.

The gradient vector of this image is then calculated at every pixel and then dotted with the vector of character- 
istic lines that the turbulent wedges propagate along. This strongly selects for the wedge structure while suppressing spurious noise and non-transition related features, such as those caused by sun/shadows on the model. A first guess is then made at the transition front by picking the location with the largest result of this dot product at each row in the image. This guess is then used to narrow the region of the image where transition is believed to be occurring. The reduced region is then re-evaluated, searching for the strongest gradient. The process is repeated until this region is collapsed into a line, which corresponds to the center of the high gradient region. This line is then used to separate the image into laminar and turbulent regions. The laminar region is then colored red, while the turbulent region is colored blue. This, when combined with the earlier mentioned green channel, results in the post processed image shown in Figure 10.

Finally, a chord scale is added to the image in black, the median transition location is marked in purple, and the median minus the median absolute deviation (MAD) of the transition location is marked in orange. All values are presented in percent chord location. This provides a robust and repeatable metric to quantify the transition location that is not subject to the bias of a user manually tracing transition fronts. Work is currently ongoing to obtain additional quantitative data from these images such as frequency content of the chordwise streaking due to the influence of crossflow vortices.

\section{Conclusion}

IR thermography provides a fast and robust method for imaging laminar-turbulent transition fronts in a wide variety of environments without perturbing the flow. The quality of the results is on par or better than the industry standard of naphthalene flow visualization, and requires only a small fraction of the time. Additionally, IR thermography responds to changing conditions in well under a second. Lastly, IR thermography data can be post processed into quantitative transition fronts.

\section{Acknowledgements}

Funding for SWIFTER is provided by the Air Force Research Laboratories (WPAFB) through General Dynamics IT. Funding for SWIFT was provided by AFOSR, the AFRL under the AEI program, and the Northrop Grumman Corporation. The authors would like to acknowledge our test pilots, Roy Martin, Dr. Donald Ward, Dr. Celine Kluzek, Lee Denham, and Lt Col Aaron Tucker; the staff of the Oran W. Nicks Low Speed Wind Tunnel, the Klebanoff-Saric Wind Tunnel, and the Texas A\&M
Flight Research Lab; and our A\&P Mechanic, Cecil Rhodes.

\section{REFERENCES}

[1] S. Zuccher and W. S. Saric, "Infrared Thermography Investigations in Transitional Supersonic Boundary Layers,” Experiments in Fluids, Vol. 44, 2008, pp. 145-157. doi:10.1007/s00348-007-0384-1

[2] W. S. Saric, H. L. Reed and D. W. Banks, "Flight Testing of Laminar Flow Control in High-Speed Boundary Layers,” The RTO Applied Vehicle Technology Panel (AVT) Specialists’ Meeting, Prague, 4-7 October 2004. http://www.cso.nato.int/Main.asp?topic=5

[3] A. L. Carpenter, W. S. Saric and H. L. Reed, "Roughness Receptivity in Swept-Wing Boundary Layers-Experiments," International Journal of Engineering Systems Modeling and Simulation, Vol. 2, No. 9, 2010, pp. 128-138. doi:10.1504/IJESMS.2010.031877

[4] D. Arnal and J. P. Archambaud, "Laminar-Turbulent Transition Control: NLF, LFC, HLFC,” Advances in Laminar-Turbulent Transition Modeling, VKI Lecture Series, Brussels, 2008.

[5] D. N. Mavris, W. S. Saric, H. Ran, M. J. Belisle, M. J. Woodruff and H. L. Reed, "Investigation of a HealthMonitoring Methodology for Future Natural Laminar Flow Transport Aircraft,” ICAS Paper 1.9.3, Nice, 2010.

[6] K. L. Chapman, M. S. Reibert, W. S. Saric and M. N. Glauser, "Boundary-Layer Transition Detection and Structure Identification through Surface Sheer-Stress Measurements," Proceedings of the 36th AIAA Aerospace Sciences Meeting and Exhibit, Reno, 12-15 January 1998.

[7] A. Ahmed, W. H. Wentz Jr. and R. Nyenhuis, "Natural Laminar Flow Flight Experiments on a Swept-Wing Business Jet," Proceedings of the AIAA 2nd Applied Aerodynamics Conference, Seattle, 21-23 August 1984.

[8] A. Drake, "Oil Film Interferometry for Boundary Layer Measurements in Aircraft Development," Proceedings of the 24th AIAA Aerodynamic Measurement Technology and Ground Testing Conference, Portland, 28 June-1 July 2004. doi:10.2514/6.2004-2114

[9] M. McQuilling, M. Wolff, S. Fonov, J. Crafton and R. Sondergaard, "An Experimental Investigation of LowPressure Turbine Blade Suction Surface Stresses Using S3F," Proceedings of the 44th AIAA Aerospace Sciences Meeting and Exhibit, Reno, 2006.

[10] A. L. Carpenter, "In-Flight Receptivity Experiments on a 30-Degree Swept-Wing Using Micron-Sized Discrete Roughness Elements,” Ph.D. Thesis, Texas A\&M University, College Station, 2009.

[11] G. T. Duncan Jr., B. K. Crawford and W. S. Saric, "Flight Experiments on the Effects of Step Excrescences on Swept-Wing Transition,” Proceedings of the 48th Applied Aerodynamics Symposium, Saint-Louis, 25-27 March 2013. 